\title{
Pinholes may Mimic Tunneling
}

\author{
D.A. Rabson \\ Department of Physics, PHY 114, University of South Florida, Tampa, FL 33620, USA \\ B.J. Jönsson-Åkerman, A.H. Romero, ${ }^{a}$ R. Escudero, C. Leighton, S. Kim, Ivan K. Schuller \\ Physics Department 0319, University of California, San Diego, La Jolla, CA 92093, USA
}

Interest in magnetic-tunnel junctions has prompted a re-examination of tunneling measurements through thin insulating films. In any study of metal-insulator-metal trilayers, one tries to eliminate the possibility of pinholes (small areas over which the thickness of the insulator goes to zero so that the upper and lower metals of the trilayer make direct contact). Recently, we have presented experimental evidence that ferromagnet-insulator-normal trilayers that appear from current-voltage plots to be pinhole-free may nonetheless in some cases harbor pinholes. Here, we show how pinholes may arise in a simple but realistic model of film deposition and that purely classical conduction through pinholes may mimic one aspect of tunneling, the exponential decay in current with insulating thickness.

${ }^{a}$ Current address: Max-Planck Institut, Heisenbergstraße 1, 70569 Stuttgart, Germany

\author{
Submitted to Journal of Applied Physics
}




\section{Tunnel Junctions}

The construction of magnetic tunnel junctions with large room-temperature magnetoresistance $^{1}$ has triggered an intense research effort, as groups have applied them as magneticfield sensors, ${ }^{2}$ memory devices, ${ }^{3-4}$ and magnetic-medium read heads. ${ }^{5}$ The current technological drive toward a lower product of resistance and area, ${ }^{6-7}$ which implies the use of thinner and thinner insulating barriers, has re-opened the question of how to rule out the presence of pinholes, direct metal-metal contacts through the nominally insulating barrier. Recent high magnetoresistances of up to $300 \%$ at room temperature observed in magnetic nanocontacts ${ }^{8}$ raise the intriguing question of whether conduction through pinholes might actually contribute to the magnetoresistance of tunnel junctions. Similarly, any study of anomalous capacitance of magnetic tunnel junctions ${ }^{9-10}$ must take pinholes into account. To exclude the possibility of pinholes, Rowell and others in the 1960s and 1970s developed a set of criteria to distinguish tunneling from other current paths. ${ }^{11}$ Four of these criteria continue to apply when neither electrode superconducts: (1) the exponential thickness dependence of the current, (2) the parabolic shape of the differential conductance as a function of voltage, (3) the scaling of the junction resistance with area, and (4) the temperature dependence of the conductivity.

We have recently constructed a series of junctions demonstrating experimentally that the second criterion applied alone cannot distinguish pinhole conduction from tunneling. ${ }^{12-13}$ The present work will show the first criterion similarly unreliable, since a purely classical conduction path through pinholes may under realistic assumptions mimic the exponential thickness dependence of the conduction current. This leaves only the temperature and area dependences of conductivity as good criteria for determining whether a junction contains pinholes.

Kleinsasser et al. ${ }^{14}$ have shown that another of the proposed signatures of tunneling in superconducting-insulator-normal junctions, the subharmonic gap structure of Andreev reflection, may similarly reflect pinhole conduction, but the problem of flushing out pinholes from normal-metal junctions remains.

Small pinholes may be invisible to surface microscopy, although recently "hot spots" have been observed using scanning tunneling microscopy. ${ }^{15}$ Moreover, the large resistivity ratio between an insulator and a metal (about $10^{6}-10^{8}$ ) requires that, over the junction area, pinhole regions of one part in $10^{6}-10^{8}$ must be ruled out to ensure no pinhole conduction in parallel with tunneling. Generally speaking, this is very difficult to do and not commonly considered. Recognizing the technological, as well as scientific, importance of identifying pinholes in magnetic tunnel junctions, Schad et al. have developed a method for imaging pinholes through decoration by electrodeposited copper. ${ }^{16}$ This tool complements criterion (4) of temperature dependence and further highlights the risks of relying solely on thickness dependence and differential conductance.

\section{Tunneling versus Pinhole Conduction}

In a series of metal-insulator-metal trilayers in which the insulating thickness, $z$, varies, the current at given voltage should decay exponentially in $z$ (the applied voltage is assumed less than the insulating gap). Such exponential decay has been cited as experimental evidence for good tunnel junctions. ${ }^{17}$ By the WKB approximation, the characteristic decay length 
equals

$$
z_{0}=\frac{\hbar}{2(2 m \phi)^{1 / 2}}
$$

where $m$ is an effective mass and $\phi$ a potential energy on the order of the barrier height. For $\phi=1 / 4 \mathrm{eV}$ and $m$ the bare electron mass, $z_{0} \sim 2 \AA$. Notably, for realistic parameters $\phi$ and $m$, this decay length coincides roughly with the thickness of a single atomic layer. In contrast, a classical resistor supports a current inversely proportional to the thickness.

The inverse relationship holds for a perfectly even layer, but real deposition processes leave an uneven insulator with possible pinholes. The simplest model for classical conduction by pinholes gives an exponential dependence of resistance on deposited thickness, mimicking quantum tunneling. Consider a metallic substrate on top of which we randomly deposit cubes of a perfect insulator to an average height $\mu$ (measured in monolayers). This is not a uniform height, so there may be pinholes. We then deposit a perfect conductor, making contact through any pinholes with the metal substrate. Since the insulator and overlayer are perfect, conduction is directly proportional to contact area and inversely to the metal-metal contact resistance, $R_{0}$.

We consider deposition to take place on a regular two-dimensional grid, each cell of which may be occupied by any non-negative integral height $(0,1, \ldots)$ of insulating particles. If each deposited particle can land randomly over any grid cell, the resulting heights follow a Poisson distribution. In the limit of an infinite two-dimensional grid, the probability that any given grid cell contains no insulating particles is $\exp (-\mu)$. For a large system, this is also the expected proportion of cells that will be unoccupied and so proportional to the conduction. Thus where tunneling can lead to an exponential-decay length of a monolayer for certain realistic parameters, the simple classical model always gives a decay length of one layer.

This trivial model (perfect insulating blocks) predicts conductance that decays exponentially in coverage $\mu$ for all coverages. However, one expects a crossover to $1 / \mu$ decay at larger coverages. The simplest extension of the model that might display such behavior deposits blocks randomly, but the insulators now have some finite resistance, $R$. In the regime of interest, $R \gg R_{0}$. As before, we deposit $M$ blocks over an $L \times L$ lattice for a coverage $\mu=M / L^{2}$ (with length measured in units of monolayer thickness). A face between the metal overlayer and an insulating block has resistance $R$, as does a face between two insulating blocks. Any connection to ground has contact resistance $R_{0}$. To make a simple model, we first turn off sideways conduction in the insulators, reducing the problem to that of an ensemble of independent columns of binomially-distributed resistors in parallel. We compare this "independent-column" model to numerical calculations below. Finally, we shall consider numerically an isotropic model that realistically models growth.

\section{Independent-column model}

Each independent column contains $n$ resistors of value $R$, where $n$ is the column height, and one contact resistor of value $R_{0}$. Letting $s=R_{0} / R$, we have for the average column 
conductance $<\sigma>=\left\langle\frac{1}{(n+s) R}\right\rangle$. In the Poisson limit, $L^{2} \rightarrow \infty$, the scaled conductance

$$
\begin{aligned}
S=R<\sigma> & =e^{-\mu} \sum_{n=0}^{\infty} \frac{\mu^{n}}{n !(n+s)} \\
& =e^{-\mu} \mu^{-s} \int_{0}^{\mu} t^{s-1} e^{t} d t=\frac{1}{s} e^{-\mu}{ }_{1} F_{1}(s, s+1 ; \mu)
\end{aligned}
$$

Here, ${ }_{1} F_{1}$ is a confluent hypergeometric function.

One arrives at an asymptotic series for large $\mu$ through successive integrations by parts, ${ }^{18}$ exhibiting explicitly the approach to $1 / \mu$ conductance:

$$
R<\sigma>\sim \mu^{-1}\left[1+\Gamma(s) \sum_{j=1}^{n} \frac{(-1)^{j}}{\Gamma(s-j) \mu^{j}}\right]
$$

where $n$ is a cutoff that must be introduced for any finite $\mu$.

More interesting is the small- $\mu$ limit, in which we recover the exponential decay of the trivial model, for multiplying the top equation in (2) by $s$, we have

$$
R_{0}<\sigma>=e^{-\mu}\left(1+s \sum_{n=1}^{\infty} \frac{\mu^{n}}{n !(n+s)}\right)
$$

For small enough $\mu, R_{0}<\sigma>\sim e^{-\mu}$, representing exponential decay of conductance with coverage.

We can estimate the crossover scale $\mu_{0}$ above which the decay ceases to be exponential by setting the " 1 " term in parentheses in (4) equal to the remainder. This yields the condition

$$
2={ }_{1} F_{1}\left(s, s+1 ; \mu_{0}\right) \quad .
$$

For $s \lesssim 0.5$, we can replace ${ }_{1} F_{1}$ with the leading term in its asymptotic expansion, giving

$$
\mu_{0} \approx \ln (2 / s)
$$

The logarithmic form of (6) comes as something of a surprise: it means that the non-zero contact resistance cuts off the small-coverage, exponential regime no matter how tiny $R_{0}$ is relative to the insulating $R$.

The solid traces (for different $s$ ) in the semilog plot, figure 1, clearly show the exponential regime for small $\mu$ and the gradual deviation as pinhole conduction ceases to dominate. To display large- and small- $\mu$ behaviors together, it is helpful to plot $y=d \ln S / d \ln \mu=S^{\prime} \mu / S$ against $\mu$; the exponential regime is characterized by a constant slope, while in the asymptotic $1 / \mu$ regime, the graph approaches the constant $y=-1$ (inset to figure 1 ). In this independent-column model, the minimum of the graph corresponds closely to the estimate (6); later, in numerical simulations incorporating surface relaxation, we see deviations somewhat earlier.

We have assumed the ratio $s$ small, as supported by the following estimate. Using Sharvin's semiclassical calculation ${ }^{19}$ for contact resistance through a pinhole of diameter 


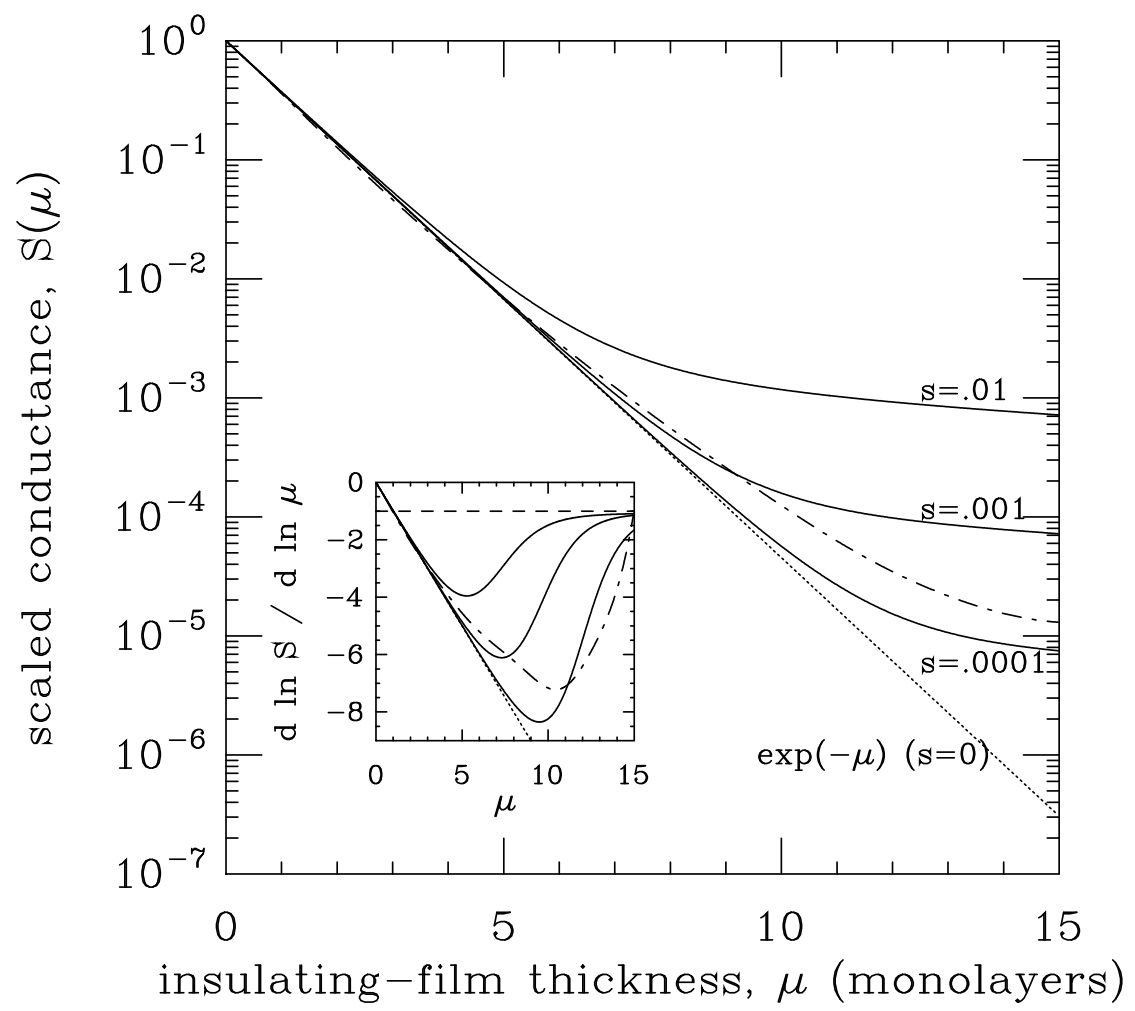

FIGURE 1. Scaled conductances (solid traces) given by (2) for three values of the ratio $s$ of contact to insulating resistance. According to the estimate (6), they begin to cross over from exponential to inverse-thickness behavior at about 5.3, 7.6, and 9.9. For comparison, we define the heuristic measure $\mu_{15}$ as the point at which the slope deviates from -1 by $15 \%$; the corresponding estimates are $4.4,7.2$, and 9.8 . The dotted line $(s=0)$ is an exact exponential. The dot-dashed line shows a numerical simulation (100000 trials on $5 \times 5$ grids) for $s=0.0001$ with $J=-0.5$, diffusion $x=1 / 3$, and "up"-steps allowed. The inset plots the same curves in a way that makes explicit the approach to $1 / \mu$ conductance $(d \ln S / d \ln \mu=-1$, horizontal line) as well as the initial exponential regime. Note that surface relaxation in this case causes a more rapid deviation from exponential decay $\left(\mu_{15}=6.1\right)$ while also delaying the onset of $1 / \mu$ conductance.

$\sim 1 \AA$, and assuming a typical metallic Fermi temperature and electronic density, we have contact resistance $R_{0} \sim 10^{4} \Omega$. The "classical" resistance of an insulating block is less well defined, but a minimum resistivity $\sim 10^{6} \Omega$ cm suggests $R \gtrsim 10^{14} \Omega$, so that $s=R_{0} / R \lesssim 10^{-10}$, tiny indeed. We then estimate by (6) that insulating thicknesses up to about 24 monolayers should show exponential resistance.

\section{Numerical Simulations}

The preceding model provides a baseline for our simulations. In addition to restoring conduction through the sides of blocks, we adopt a model due to Pal and Landau ${ }^{20-22}$ to describe the motion of insulating blocks along the surface after they have fallen. Such models have found good agreement with experiment for long-wavelength and long-time (large- $\mu$ ) features, although our interest lies in the opposite regime.

For simplicity, we imagine the original metallic layer to be flat. ${ }^{23}$ After depositing some number of blocks in the manner of the previous section, we allow a fraction of blocks at the interface to diffuse one unit along the surface. We employ a quasi-Metropolis procedure to determine whether to accept or reject each move (the system is not at equilibrium) with an energy $J$ for each face-to-face bond between blocks: calling $D$ the change in the number of bonds, the move is accepted unconditionally if it lowers the energy and otherwise with 
probability $\exp \left(-D J / k_{\mathrm{B}} T\right)$. Henceforth, we scale $J$ by setting the thermal energy $k_{\mathrm{B}} T=1$. Thus a negative $J$ encourages clustering, while a positive $J$ could represent stearic hindrance or an affinity for roughness. We adopt one more feature from the Pal-Landau models: blocks either may or may not jump "up" (further away from the substrate) while relaxing. ${ }^{22}$

We perform the simulation on an $L \times L$ grid, measuring conductance at set times, before averaging over many trials to reduce noise. Since long-wavelength features do not concern us in the present work, $L$ can be as small as allowed by diffusion. Consider the diffusion parameter $x$ defined as the fraction of interface blocks diffusing one lattice spacing per monolayer deposited. A simple random-walk argument establishes that for $x$ of order unity, we can look at coverages as great as about 25 for a $5 \times 5$ grid. Numerically, we tested values of $L$ from 5 to 60 and found no significant differences in conductances. The actual diffusion parameter $x$ of course will depend on temperature and physisorption and chemisorption energies. We considered diffusion parameters $x=0.33-10$, roughly corresponding to a fast, but not unrealistic, deposition rate. ${ }^{24}$

This model must behave the same as the independent columns in the two limits of zero and large coverage, but as figure 1 shows, surface relaxation can reduce the thickness at which deviations from exponential decay first become apparent, primarily by modifying the rate at which pinholes fill in. Because the deviation occurs continuously, we need a heuristic measure. The slope of the line in a semilog plot (figure 1) determines the decay length; in both the simple model and the simulations, it always starts out at -1 . Such a plot appears to differ by eye from a straight line when the slope changes by $15 \%$; we call this crossover scale $\mu_{15}$. As noted in the caption to figure 1 , it also agrees reasonably well with the analytical crossover estimate (6) for the independent-column model with no relaxation.

Figure 2 plots $\mu_{15}$ as a function of bond strength, $J$, for a few selected values of $s$ and the relaxation parameter, $x$. We find that relaxation still permits a large value for $\mu_{15}$ for particular ranges of $J$. When blocks are permitted to jump up, there is a tendency for pinholes to be extinguished less quickly than exponentially (see dot-dashed curve in figure 1), since at moderate coverage more columns of height 1 turn into pinholes than vice-versa. A sufficiently attractive (negative) $J$ counters this tendency by discouraging jumps out of height-1 columns, but too large a negative value of $J$ leads to superexponential decay. In the model without jumps, a sufficiently large positive (repulsive) $J$ effectively cancels the effect of surface relaxation on pinholes.

In some cases, we find that a reasonably large negative $J$ can effectively alter the exponential-decay length over some range of coverages; see figure 3. To get a decay length substantially different from one monolayer, we need to modify the model. For example by prohibiting direct deposition on top of isolated pinholes (as by stearic hindrance), filling these only by surface relaxation, we compute both a longer decay length and a larger crossover scale (not shown).

\section{Implications for experiment}

We have concentrated on one signature of tunneling, showing that purely classical pinhole conduction can mimic the exponential dependence of resistance on barrier thickness. We have recently demonstrated experimentally that another signature, the nonlinear form of current $I(V)$ (as a function of bias $V)^{25-26}$ also may fail to distinguish classical conduction from tunneling. ${ }^{12}$ In a series of ferromagnet-insulator-metal junctions in which the "metal" is actually a superconductor, all samples could be fit well to a tunneling form above the 


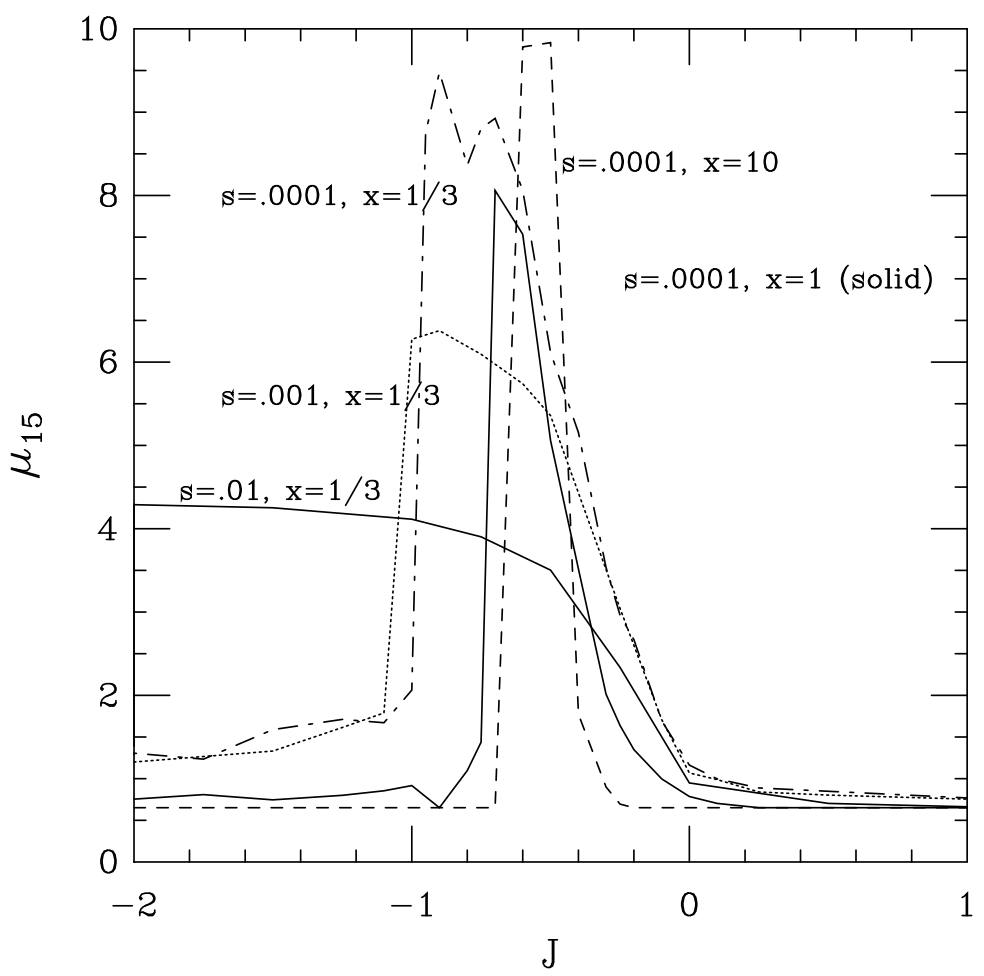

FIGURE 2. The horizontal axis measures the bond strength (negative attracts blocks), the vertical an estimated maximum thickness for the mimicry of tunneling, specifically the empirical measure $\mu_{15}$ at which the slope of a semilog plot, as in figure 1, deviates by $15 \%$ from -1 . For comparison, the cutoff estimates (6) without relaxation for $s=.01$, .001 , and .0001 are 5.3, 7.6, and 9.9. Blocks may jump up in these traces.

superconductor's transition temperature, $T_{c}$. However, some (but not all) showed Andreev reflection below $T_{c}$, indicating the presence of pinhole shorts through the insulator. ${ }^{27}$ In other words, samples that appear (according to $d I / d V$ and thickness dependence $I(z)$ ) to be good tunnel junctions may not be.

We would expect that increasing temperature should enhance current through a tunnel junction (effectively lowering the barrier) while suppressing conduction through any metallic short. In fact, we found that the temperature dependence of current $I(T)$ at zero bias does distinguish the pinhole-free from the shorted samples.

\section{Acknowledgements}

We thank H. Srikanth and M. Devoret for helpful discussions and acknowledge the coding assistance of Mr. Michael Grossman. This work was supported in part by DARPA and ONR. 


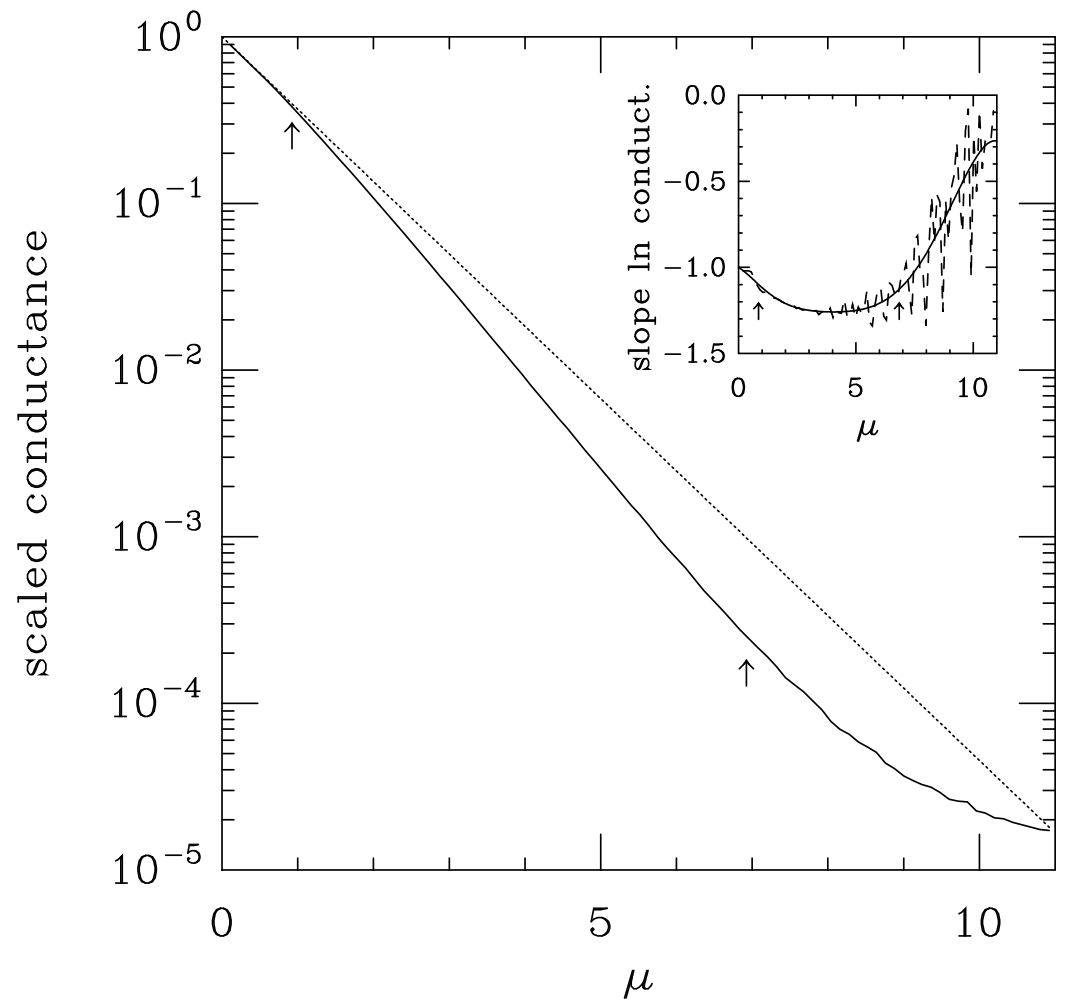

FIGURE 3. An example of a different effective slope: the dotted line is an exact exponential. Between the arrows $(\uparrow \ldots \uparrow \mu \approx 1 \ldots 7)$, it is difficult to distinguish the simulated decay with relaxation $(s=.0001, x=1 / 3$, $J=-2$ ) from a straight line of slope $\sim-1.25$, i.e., a decay length of 0.8 monolayers. No smoothing has been applied to the main graph. The inset shows the slope of the decay; the solid line applies parametric smoothing.

\section{References}

1 J.S. Moodera, L.R. Kinder, T.M. Wong, and R. Meservey, Phys. Rev. Lett. 74, 3273 (1995).

2 J.M. Daughton, J. Appl. Phys. 81, 3758 (1997).

3 S.S.P. Parkin, K.P. Roche, M.G. Samant, P.M. Rice, R.B. Beyers, R.E. Scheuerlein, E.J. O'Sullivan, S.L. Brown, J. Bucchigano, D.W. Abraham, Y. Lu, M. Rooks, P.L. Trouilloud, R.A. Wanner, and W.J. Gallagher, J. Appl. Phys. 85, 5828 (1999).

4 S. Tehrani, J.M. Slaughter, E. Chen, M. Durlam, J. Shi, and M. DeHerrera, IEEE Trans. Magn. 35, 2814 (1999).

5 J. Zhang, Data Storage 5, 31 (1998).

6 D. Song, J. Nowak, and M. Covington, J. Appl. Phys. 87, 5197 (2000).

7 E.Y. Chen, R. Whig, J.M. Slaughter, D. Cronk, J. Goggin, G. Steiner, and S. Tehrani, J. Appl. Phys. 87, 6061 (2000).

8 N. García, M. Muñoz, and Y.-W. Zhao, Phys. Rev. Lett. 82, 2923 (1999); G. Tatara, Y.-W. Zhao, M. Muñoz, and N. García, Phys. Rev. Lett. 83, 2030 (1999).

${ }^{9}$ K.T. McCarthy, S.B. Arnason, and A.F. Hebard, Appl. Phys. Lett. 74, 302 (1999).

10 S. Zhang, Phys. Rev. Lett. 83, 640 (1999).

11 See e.g. E. Burstein and S. Lundqvist, eds., Tunneling Phenomena in Solids, Plenum Press, New York, 1969. 
12 B.J. Jönsson-Åkerman, R. Escudero, C. Leighton, S. Kim, I.K. Schuller, and D.A. Rabson, to appear in Applied Physics Letters.

13 H. Srikanth and A.K. Raychaudhuri, Phys. Rev. B46, 14713 (1992) find a change in the sign of curvature of differential conductance, due to local heating, for a series of scanningtunneling point contacts ranging from microshorts to tunnel junctions. Their well-defined point contacts differ from our accidental pinholes.

14 A.W. Kleinsasser, R.E. Miller, W.H. Mallison, and G.B. Arnold, Phys. Rev. Lett. 72, 1738 (1994).

15 W. Wulfhekel, B. Heinrich, M. Klaua, T. Monchesky, F. Zavaliche, R. Urban, and J. Kirschner, "Characterization of single-crystal magnetotunnel junctions by local tunneling," unpublished (2000).

16 R. Schad, D. Allen, G. Zangari, I. Zana, D. Yang, M. Tondra, and D. Wang, Appl. Phys. Lett. 76, 607 (2000); D. Allen, R. Schad, G. Zangari, I. Zana, D. Yang, M. Tondra, and D. Wang, J. Appl. Phys. 87, 5188 (2000).

17 R. Meservey, P.M. Tedrow, and J.S. Brooks, J. Appl. Phys. 53, 1563 (1982).

18 C.M. Bender and S.A. Orszag, Advanced Mathematical Methods for Scientists and Engineers, McGraw-Hill, New York, 1978.

19 Yu. V. Sharvin, Zh. Eksp. Teor. Fiz. 48, 984 (1965) [Sov. Phys. JETP 21, 655 (1965)].

20 S. Pal and D.P. Landau, Phys. Rev. B49, 10597 (1994).

21 D.P. Landau and S. Pal, Thin Solid Films 272, 184 (1996).

22 D.P. Landau and S. Pal, Langmuir 12, 29 (1996).

23 One might also model the effect of polishing scratches in seeding pinholes by imposing a surface potential on the substrate; see D.J. Keavney, E.E. Fullerton, and S.D. Bader, J. Appl. Phys. 81, 795 (1997).

24 D.L. Smith, Thin-Film Deposition: Principles and Practice, McGraw-Hill, New York, 1995.

25 J.G. Simmons, J. Appl. Phys. 34, 1793 (1963).

26 W.F. Brinkman, R.C. Dynes, and J.M. Rowell, J. Appl. Phys. 41, 1915 (1970).

27 G.E. Blonder, M. Tinkham, and T.M. Klapwijk, Phys. Rev. B25, 4515 (1982) and G.E. Blonder and M. Tinkham, Phys. Rev. B27, 112 (1983) examine the crossover from Andreev reflection to tunneling as the insulating barrier increases in strength. As a practical matter, with a typical band gap $(\sim 10 \mathrm{eV})$, the Andreev signature should become insignificant for insulating thicknesses greater than a few monolayers. Thus, the presence of Andreev reflection indicates either a pinhole or a "near" pinhole. 